\title{
Ensayo terapéutico con Levamisole en bronquitis obstructiva recidivante
}

Drs. G. Dragicevic A., 1 న. Concha C. ${ }^{2}$ y V. Valdés L. ${ }^{3}$

\begin{abstract}
The results obtained in the treatment of 48 children bearer of infectious asthma with Levamisole in the dosis of $2,5 \mathrm{mg} / \mathrm{kg}$, twice a day, in two tween days a week, for a period of six months.

Good results abtained, similar to the ones met by means of other procedures are shown and compared to the opposit situation observed in non-treated infants.

The possible marrow frenation described with Levamisole was not observed in our infants.
\end{abstract}

Desde 1971, en atención a la elevada incidencia de Bronquitis Obstructiva Recidivante (BOR) en nuestra comuna, hemos estado ensayando diversas alternativas terapéuticas, ${ }^{3,4}$ tratando de simplificar los esquemas y basados en los antecedentes que proporciona la literatura extranjera ${ }^{7 \cdot i 0}$ sobre la utilidad y mecanismos de acción del clorhidrato de $L(-)-2,3,5,6$ - tetrahidro-6-fenilimidazo [2,1-b] tiazol (Levamisol, fig. 1), en 1979 y 1980 hemos iniciado series de estudio de la aplicabilidad del producto en la Enfermedad Bronquial Obstructiva del niño.

\section{Figura 1}

\section{PRIMCIPIO ACTIVO}

\section{Cevaminol}

FORMULA

QUIMICA

Clorhidrato de $L-(-)-2,3,5,6-$ tetrahidro-6- fonllimidaro $[2,1-b]$ tiazol.

\section{ESTRUCTURA} OUIMICA

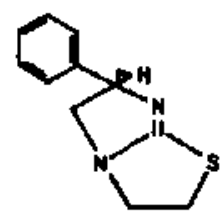

\section{PROPOSITOS}

En esta serie en análisis hacemos un informe de un ensayo terapéutico midiendo la eficacia de Levamisole en la BOR, así como estudiando y mi-

\footnotetext{
ITrabajo presentadoen las VII Jomadas de Pediatrta en Arica.

2 Pediatra - Director Hospital de Peñaflor.

3Miédicos Generales de Zona.
}

diendo uno de los riesgos del producto que sería la posibilidad de frenación medular.

\section{MATERIAL Y METODO}

En 48 niños portadores de Bronquitis Obstructiva Recidivante tratados con Levamisole en dosis de $2,5 \mathrm{mg} \times \mathrm{kg}$ de peso por dia, dividido en dos tomas diarias, en dos días seguidos semanales durante seis meses, conforme a lo recomendado ${ }^{8,} 10$ y en 10 niños que no terminaron terapia por abandono del esquema o de la vacuna microbiana en el mismo período en estudio comprendido entre octubre de 1979 y marzo de 1980 , se revisan algunas características clínicas que permitan medir el éxito o fracaso de la acción del Levamisole. En el grupo de estudio se hizo evaluación sistemática del estado hematológico para detectar frenación medular si ella se producía.

\section{RESULTADOS}

En la Tabla l se señalan las características clínicas propias del grupo de estudio, en los parámetros que hacen más objetiva la evolución del cuadro.

\section{Tabla 1}

Caracteristicas de iniciación, frecuencia y duración de los síntomas en BOR en el grupo estudiado

Edad de comienzo sintomas:

$\begin{array}{lcr}0-3 \text { meses } & 20 & 36,36 \% \\ 3-6 \text { meses } & 19 & 34,55 \% \\ 6-12 \text { meses } & 11 & 20,00 \% \\ 12 \text { meses o más } & 5 & 9,00 \% \\ & \overline{55 \text { niños }} & 100 \%\end{array}$


Semanal

Quincenal

Mensual

Permanente

Total

Duración de los sintomas:

Menor de 7 días

+ de 7 y - de 10 días

10 días $y$ más

Total

\begin{tabular}{lr}
2 & $4 \%$ \\
27 & $54 \%$ \\
18 & $36 \%$ \\
3 & $5 \%$ \\
\hline 50 niños & $100 \%$
\end{tabular}

27

15

11

5.3 niños
- Antes del tratamiento : 14

- Después de iniciado el

tratamiento

En la Tabla 3 destacamos la evolución de un grupo pequeño de control.

\section{Tabla I}

Evolución en 10 niños que no completaron sus tratamientos con autovacuna o Levamisole

A) Apreciación subjetica:

$\begin{array}{rrrr}- \text { Igual } & : & 8 & (80 \%) \\ - \text { Bien } & : & 2 & *\{20 \%) \\ \text { Total } & & 10 & \end{array}$

B) Reagudizaciones:

$$
\begin{array}{lrrr}
\text { - Ninguna } & : & 1 & (10 \%) \\
-20 \text { menos } & : & 1 & (10 \%) \\
\text { - llas de 2 } & 8 & (80 \%) \\
\text { Total } & -10 & \\
&
\end{array}
$$

En la Tabla 4 se expresa de manera simplificada un análisis de la evaluación hematologica sistemática resumida en los valores previos y finales

\begin{tabular}{|c|c|c|c|c|}
\hline & Sormal & Anemio & Total & \\
\hline Inicial & $42(91,30 \%)$ & $4(8,8 \%)$ & 46 & \\
\hline Final & $39(95,12 \%)$ & $2(4,88 \%)$ & 41 & \\
\hline LELCOCITOS & Normal & Letucocitosis & Total & \\
\hline Inicial & $44(95,65 \%)$ & $2(4,35 \%)$ & 46 & \\
\hline Final & $37(90,24 \%)$ & $4\langle 9,76 \%)$ & 41 & \\
\hline LNAFOCITOS & Normales & Aumentados & Disminuidos & Total \\
\hline Inicial & $8(16,40 \%)$ & $2 L(45,65 \%)$ & $17(37,95 \%)$ & 46 \\
\hline Final & $8(19,52 \%)$ & $16(39,02 \%)$ & $17(41,46 \%)$ & 41 \\
\hline
\end{tabular}
de algunos parámetros del hemograma.

\section{Tabla 4}

Evaluación hematológica previa y final en el tratamiento de la BOR con Levamisole

A) HEMATOCRITO

Vota: Sin diferencias estadisticamente significativas en ninguin caso. 


\section{DISCUSION Y COMENTARIO}

La dosis útil y no tóxica recomendada por la literatura para el uso de Levamisole es de $2,5 \mathrm{mg} \times \mathrm{kg} \times$ día, dividido en dos tomas diarias, en dos días seguidos semanales durante seis meses; ${ }^{8 .}{ }^{10} \mathrm{con}$ este esquema terapéutico logramos obtener una mejoría en la evolución en la totalidad de los casos según apreciación de la madre; la ausencia de fracasos la atribuinos obviamente al tamaño de la muestra; avalando la tendencia medida por el procedimiento anterior está el resultado en cuanto a reagudizaciones que en la historia natural del cuadro se producen en un $90 \%$ quincenal o mensualmente y con el Levamisole no ofrece recidivas en un $64,58 \%$ y de 2 o menos en 6 meses en un $29,17 \%$, o sea una situación totalmente opuesta.

En el $29 \%$ de los casos previo al tratamiento se requirió hospitalización de los niños, no siendo necesario en ningún caso durante el periodo de terapia con Levarnisole.

Contrariamente a lo señalado en el grupo tratado, cuando analizamos un grupo de control apreciamoṣ que siguẹn su curso natural el $80 \%$ de los casos.

Otro de los factores que a nuestro juicio explican los resultados obtenidos en niños tratados con inmunoestimulantes o inmunorreguladores es la capacidad reaccional del sistema inmunocompetente y que también puede explicar la diferencia de apreciación existente entre los resultados obtenidos con vacuna microbiana en bronquíticos crónicos en adultos y los conseguidos con el mis- mo procedimiento en dosis y duración adecuados en niños. $1.2,3,6$

Como uno de los problemas descritos en la literatura sobre el uso de Levamisole fue el de la frenación medular, hicimos evaluación sistematica del estado hematológico de los pacientes; en la Tabla 4 se señala cómo se encontró el hematocrito al comienzo y al final y el número de leucocitos antes $y$ al término del tratamiento como una muestra de lo que encontrábamos.

En la serie roja el hematocrito no reveló variaciones estadísticamente significativas durante el tratamiento y en la serie blanca se observa la misma situación.

En consideración a que el posible mecanismo de acción fisiopatológico fuera a través de los linfocitos, se tabuló el recuento de la línfocitosis relativa, no existiendo variaciones significativas entre el comienzo y el final del estudio.

En este momento se encueptia en ejecución un trabajo cooperativo con el' La' oratorio de Inmunología de la Facultad de Medicina Sede Occidente de la Universidarl de rlible, en que se está efectuando estudio de rosetas y medición de inmunoglobulinas como método de cuantificar la acción del Levamisole y eshudiar su posible mecanismo de acción a través de una regulación del equilibrio entre linfocito $T$ supresor y la producción de inmunoglobulinas protectoras en el tratamjento de la BOR y disminución de reaginas en la atopia, como sería la situación del asma bronquial tipo I.

En la Figura 2 mostramos un modelo adaptado" de la interacción de los linfocitos T y B y que

Figura 2

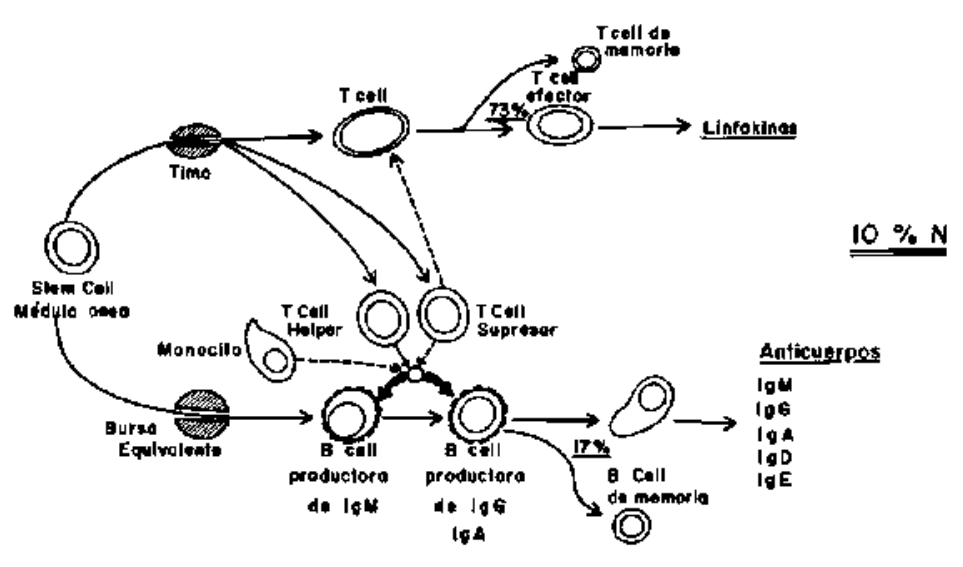

Interacclón colular involucrada en la regulactón de la síntegis de inmunoglobulinas duronte we reapuesto inmune, y lineo de la Inmunidad colvlar. 


\section{CONCENTRACION DE INMUNOGLOBULINAS}

CURVA ONTOGENIA

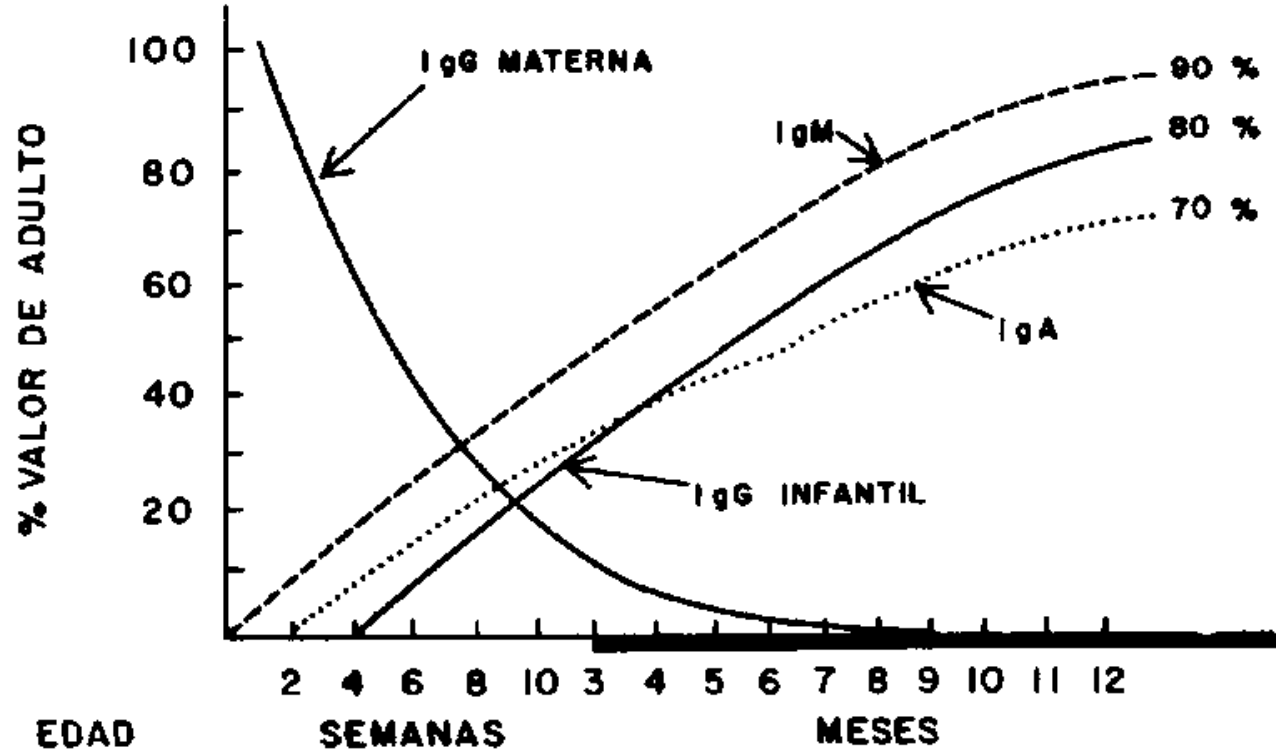

explica tambièn la curva ontogénica de producción de inmunoglobulinas de la Figura 3.

En la Figura 4 desarrollamos el esquema por el cual se regula el lumen en el bronquio en la Enfermedad Bronquial Obstnuctiva en general como ejemplo, pero que también ocurriría en los linfocitos B en el caso de la BOR o en los linfocitos T supresores en el caso del asma atópico, en que por una interconversion de los receptores beta a alfa se producirian las alteraciones propias de esos cuadros $y$ de la atopia en general, según Szentivangy. Esta interconversión podría ser corregida por el uso de Levamisole por un mecanismo similar a como actúan los corticoides, con la salvedad de que en el uso de esteroides se mantiene la reversibilidad del defecto, en cambio con el Levamisole se produce una estabilización de la situacion.

En la literatura ${ }^{7.8 .}$ se señalan diversos hallazgos tanto in vitro como in vivo sobre la acción del Levamisole que aqui enumeramos en resumen:

1. Timomimético que restablece la normalidad de la función de fagocitos y de linfocitos $\mathrm{T}$.

2. Aumenta la migración de los fagocitos y la quimiotaxis en PMN y monocitos con movilidad defectuosa.

3. Restituye la inhibición de la migración de los leucocitos en el individuo anérgico.

Figura 4

\section{ESQUEMA DINAMICA BRONOUIAL}

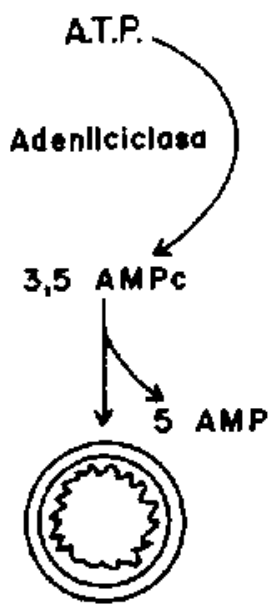

\section{Broncodilatación}

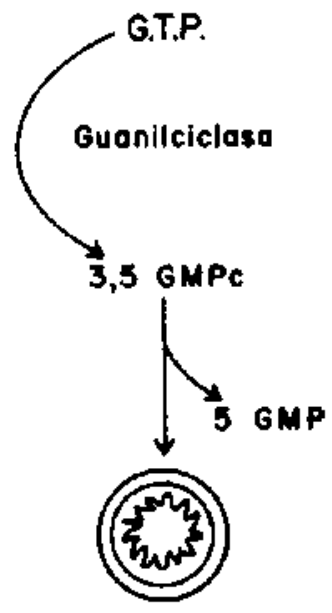

\section{Broncoconstricción}

4. Aumenta la producción de linfokinas (MAF y MIF) en presencia de mitógenos ín vitro.

5. Aumento de síntesis proteica y de ácido nucleico en linfocitos.

6. Disminución o supresión de la actividad supresora sobre linfocitos.

7. Aumento de la actividad lisosomal, peroxisomal y de ATPasa de los linfocitos. 
8. Restablece la celularidad nomal del linfocito $\mathrm{T}$ (Roseta $\mathrm{E}$ ) en los pacientes que tienen disminución de ella; cuando se acompaña de células ligadas a Ig, células plasmáticas o células formadoras de rosetas EAC aumentadas, se produce disminución de linfocitos $\mathrm{B}$ o de las células plasmáticas.

9. Aumenta el interferón in vitro pero no in vivo.

10. Aumenta concentración de cGMP (ddisminución de consumo por inversión de receptores?) y disminuye el cAMP ( $\mathrm{c}$ aumento de consumo por inversión de receptores?).

Finalmente destacamos que en la literatura en 3.046 casos estudiados por Symoens y Rosenthal, ${ }^{8}$ los efectos adversos que se han encontrado son los siguientes: sintomas gastrointestinales y del SNC o semejantes a resfrío en menos de un $5 \%$, rash cutáneo en un $2 \%$, granulocitopenia transitoria en un $0,7 \%$ y trombocitopenia en un $0,03 \%$ de los casos. Al respecto cabe señalar que cuando los controles hematológicos coincidieron con la proximidad inmediata anterior de la ingestión del producto, se apreciaron bajas en las series hematológicas, que se recuperaron rápidamente en el curso de la semana.

\section{RESUMEN}

En este artículo informamos de los resultados obtenidos en el tratamiento de $\mathbf{4 8}$ niños portadores de Bronquitis Obstructiva Recidivante en un ensayo efectuado con el producto Levamisole en la dosis recomendada en la literatura de $2,5 \mathrm{mg} \times \mathbf{~ k g}$ de peso por día, dividido en dos tomas diarias, dos días seguidos en la semana durante seis meses de tratamiento.

Los buenos resultados obtenidos, similares a los conseguidos con otros procedimientos, ${ }^{1,2.3,6}$ se comparan con la situación inversa ocurrida en niños no tratados.

Se mide el posible daño de frenación hematológica a nivel medular que no fue detectado en la serie estudiada. Se analizan los diversos mecanismos de acción invocados en la literatura y se señalan otros efectos adversos descritos también en la literatura revisada.

\section{REFERENCIAS}

${ }^{1}$ Berdach, R., y cols. Bronquitis Obstructiva Recidivante: Análisis clinico y evaluación terapéutica del tratamiento con stock vacuna en 130 lactantes. Terceras Jornadas Anuales de Pediatría. Punta Arenas, 1971.

${ }^{2}$ Casar, C., y cols. Bronguitis Obstructisa Recidivante del lactante. Estudio clínico, de laboratorio y tratamiento. Rev. Chil. Ped., 44: 340, 1973.

3 Dragicetic, C., y cols. Evaluación del trataniento con vacuna microbiana concentrada en niños portadores de Bronquítis Obstructiva Recidivante del lactante. Bol. Hosp. San Juan de Dios, 23: 16-20, 1973.

4Dragicevic, G., y cols. Diagnóstico diferencial y tratamięnto de la Bronquitis Obstructiva Recidivante y el asma bronquial en el niño. Bol. Hosp. San Juan de Lios, 24: 159-164, 1977.

5 Galleguillos, $F_{1}, y$ cols. Avances en el tratamiento del asma bronquial. Rev. Médica de Chile, 104: 7 I-74, 1976.

FMueller, $\boldsymbol{H}$, y cols. Hyposensitization with Bacterial Vaccine in infection Asthma. JAMA; 26, vol. 208, 1979, 1969.

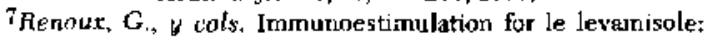
cibles et mécanismes. La Nouvelle Presse Médicale: 21 Janvier, 7, Y. 3 : 197-201, 1978.

8 Symoens, G., and fiosenthal, $M$. A Review. Levamisole in the Modulation of the Immune Response: The cument Experimeptal und Clinical State Journal of the Reticuloendothelial Society. 21, N. ${ }^{\circ} 3:$ 175-221, $197 \overline{7}$.

${ }^{9}$ Szentitingy, A. Analisis experimental de la teoria beta-adrenérgica en las enfermedades atópicas. Acta Médica FAB. 2: $11-16,1980$.

10 Van Eygen, $M$, y cols. Levamisole in prevention of recurrent upper respiratory - tract infections in children. The Lancet, 1 (7956): 382-385, 1976.

11 Watlmann, Th. A. Disorders of suppressor cells in the pathogenesis of immunodeficiency, autoimmune and allergic diseases: human disease associated with disorders of an immunological hreaking system. Annals of allergy, 39, N. ${ }^{\circ}$; 79.86, 1977 . 